\title{
Clostridium difficile Infection Associated with Antituberculous Agents in a Patient with Tuberculous Pericarditis
}

\author{
Yi-Xuan Sun ${ }^{1}$, Yin-Tao Zhao ${ }^{2}$, Li-Li Teng ${ }^{1}$, Jian-Li Ge ${ }^{1}$, Hua Jiang ${ }^{1}$ and Li Shao ${ }^{1}$
}

\begin{abstract}
Clostridium difficile can cause pseudomembranous colitis (PMC). Antimicrobial agent exposure is a risk factor for Clostridium difficile-associated disease, whereas the use of antituberculous (anti-TB) agents is not. We herein report a case of PMC-associated with antituberculous therapy. A 63-year-old woman with tuberculous pericarditis treated with anti-TB agents was admitted for abdominal pain and diarrhea. On colonoscopy, mucoid exudate and yellowish plaque lesions were observed. The anti-TB agents were discontinued, and the patient was treated with metronidazole and clostridium butyricum. Her symptoms were relieved and did not recur when the anti-TB agents were restarted. In this report, we review the literature and discuss the pathogenesis, clinical manifestations, diagnosis and treatment of this case.
\end{abstract}

Key words: Clostridium difficile, tuberculous pericarditis, antituberculous agents, diarrhea, pseudomembranous colitis

(Intern Med 52: 1495-1497, 2013)

(DOI: 10.2169/internalmedicine.52.0162)

\section{Introduction}

Clostridium difficile (C. difficile) infection is a common problem in health care that causes significant morbidity and associated mortality, especially in older persons. C. difficileassociated disease (CDAD) varies from asymptomatic colonization to mild diarrhea to severe life-threatening pseudomembranous colitis (PMC). This organism is the causative pathogen in $20-30 \%$ of cases of antibiotic-associated diarrhea and nearly all cases of antibiotic-associated colitis $(1,2)$. C. difficile can cause diseases of the gastrointestinal tract, ranging from asymptomatic colonization to the life-threatening condition known as "toxic megacolon." Nearly all classes of antimicrobials can induce CDAD, particularly clindamycin, third-generation cephalosporins, broad-spectrum penicillins and fluoroquinolones (3). However, antituberculous (anti-TB) agents are rarely associated with CDAD. We herein report a case of anti-TB agentassociated PMC. Such cases may escape the notice of clini- cians.

\section{Case Report}

A 63-year-old rural woman was admitted to our hospital due to abdominal pain and diarrhea. Pulmonary TB had been diagnosed 30 years earlier, and tuberculous pericarditis was observed in May 2009. The patient's medications included anti-TB therapy with rifampin (450 mg/day), isoniazid (300 mg/day), ethambutol (750 mg/day) and pyrazinamide (1,500 mg/day). No specific adverse drug events developed after the initiation of the anti-TB treatment.

However, severe mucoid diarrhea with abdominal cramping pain and tenesmus developed in October 2009 (four months after the initiation of anti-TB treatment). On this admission, the patient's blood pressure was $135 / 70 \mathrm{mmHg}$, her pulse rate was 104 beats/min, her respiratory rate was 17 breaths $/ \mathrm{min}$ and her body temperature was $37.2^{\circ} \mathrm{C}$. Her abdomen was soft throughout the abdominal area and her bowel sounds were hyperactive. The laboratory data ob-

${ }^{1}$ Department of Geriatric Medicine, East Hospital, Tongji University School of Medicine, China and ${ }^{2}$ Department of Cardiology, the First Affiliated Hospital of Zhengzhou University, China

Received for publication January 18, 2013; Accepted for publication March 10, 2013

Correspondence to Dr. Li Shao, dongfangshaoli@126.com 


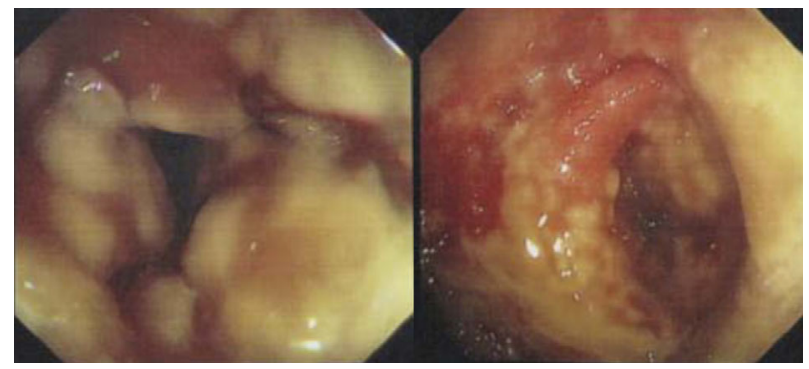

Figure 1. The initial colonoscopic examination showing mucoid exudate and yellowish plaque lesions.

tained on admission were as follows: hemoglobin: $11.7 \mathrm{~g} /$ $\mathrm{dL}$, hematocrit: $32.9 \%$, white blood cell count: $14,000 / \mu \mathrm{L}$ with $68.7 \%$ segmented neutrophils, platelets: $152,000 / \mu \mathrm{L}$, ESR: $3 \mathrm{~mm} / \mathrm{h}$ and CRP: $28.56 \mathrm{mg} / \mathrm{dL}$. Tests regarding the hepatic and renal function and serum electrolytes were close to the normal reference ranges.

The patient had no known history of hepatitis, chronic renal disease, smoking or alcohol consumption or exposure to contaminated water. Her mother was healthy and her father had died three years earlier due to an asthmatic attack. Her daughter and husband were healthy.

Initially, we considered that the patient may have bacterial enteritis. The symptoms were persistent, despite symptomatic management with oral anti-cholinergic agents and fluoroquinolone. Therefore, we withheld further anti-TB and fluoroquinolone treatment and referred the patient to a gastroenterology specialist. She then received a colonoscopic examination, which showed mucoid exudate and yellowish plaque lesions, and PMC was diagnosed (Fig. 1). In addition, a fecal culture of the patient was negative. Limited by the conditions of our hospital, we sent the sample to other hospital for Toxin A and Toxin B testing. The results, which were positive, were received after the patient was discharged from our hospital.

The patient was treated with metronidazole $(200 \mathrm{mg}$, three times per day) and clostridium butyricum for one week. Her symptoms were relieved and did not recur when the anti-TB agents were administered again. The white blood cell count was $9,000 / \mu \mathrm{L}$, the segmented neutrophil proportion was $61.9 \%$ and the CRP level was $7 \mathrm{mg} / \mathrm{dL}$. The severity of pseudomembranous colitis was found to be significantly alleviated on repeat colonoscopy (Fig. 2).

\section{Discussion}

Since the first description of $C$. difficile by Hall and O'Toole in 1935, this organism has been recognized to be the causative agent of nosocomial diarrhea. In addition, $C$. difficile was identified to be the nearly exclusive cause of PMC in 1978 (4). At present, C. difficile is the cause of approximately $25 \%$ of all cases of antibiotic-associated diarrhea worldwide (1). The most common inducing agents are clindamycin and broad-spectrum cephalosporins: however, nearly all agents with an antibacterial spectrum can cause

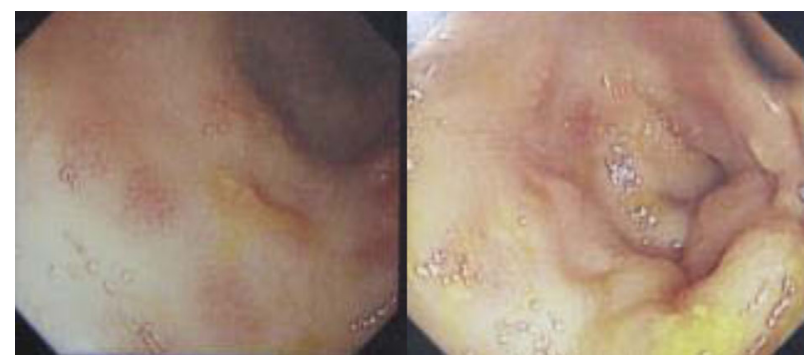

Figure 2. A repeat colonoscopic examination showing that the severity of the pseudomembranous colitis was significantly alleviated.

diarrhea. In contrast, anti-TB agents are rarely associated with this disorder. Among anti-TB agents, rifampin is postulated to be the cause of PMC because isoniazid and ethambutol have little or no effect on the intestinal flora, while rifampicin has an antibiotic effect on a wide range of bacteria (5). In our case, the patient had taken anti-TB therapy, including rifampin, isoniazid, ethambutol and pyrazinamide, for a long time due to tuberculous pericarditis; therefore, the former drug is most likely the cause of the PMC observed in this case.

C. difficile is spread by the fecal-oral route and can be ingested in a vegetative or spore form. The spore form of the bacterium is extremely durable, allowing the pathogen to survive not only harsh conditions outside of the human body, but also the acidic environment of the stomach. After ingestion, spores germinate in the vegetative form in the small intestines. One of the most common causes of disruption of the normal gastrointestinal flora is the use of antimicrobial agents, particularly broad-spectrum agents with antianaerobic activity.

It is important to note here that clinicians should use Toxin A and Toxin B tests to diagnose PMC. We hope to improve our medical facilities in the future.

\section{The authors state that they have no Conflict of Interest (COI).}

\section{Acknowledgement}

This research was supported by grants from the National Natural Science Foundation of China (30600294), Natural Science Foundation of Shanghai (11ZR1429500), Key Disciplines Group Construction Project of Pudong Health Bureau of Shanghai (PWZxkq2010-05) Academic Leaders Training Program of Pudong Health Bureau of Shanghai (PWRd2011-01) and the Science and Technology Development Fund of Pudong New district (PKJ2012-Y07).

Yi-Xuan Sun and Yin-Tao Zhao contributed equally to this work.

\section{References}

1. Bartlett JG. Antibiotic-associated diarrhea. Clin Infect Dis 15: 573-581, 1992.

2. Bouza E, Burillo A, Muñoz P. Antimicrobial therapy of Clostrid- 
Intern Med 52: 1495-1497, 2013 DOI: 10.2169/internalmedicine.52.0162

ium difficile-associated diarrhea. Med Clin North Am 90: 1141$1163,2006$.

3. O'Keefe SJ. Tube feeding, the microbiota, and Clostridium difficile infection. World J Gastroenterol 16: 139-142, 2010.

4. Bartlett JG, Chang TW, Gurwith M, Gorbach SL, Onderdonk AB.
Antibiotic-associated pseudomembranous colitis due to toxinproducing clostridia. N Engl J Med 298: 531-534, 1978.

5. Jung SW, Jeon SW, Do BH, et al. Clinical aspects of rifampicinassociated pseudomembranous colitis. J Clin Gastroenterol 41: 3840, 2007.

(C) 2013 The Japanese Society of Internal Medicine http://www.naika.or.jp/imonline/index.html 